\title{
La Merveilleuse Aventure de Marius Barbeau. Jacques Cartier sous l'éclairage de Bougainville
}

\author{
Lucie Robert \\ Université du Québec à Montréal
}

\begin{abstract}
Résumé
En 1934, l'année même où l'on célèbre le $400^{\mathrm{e}}$ anniversaire de la découverte du Canada, Marius Barbeau, déjà connu pour son travail d'ethnologue, publie un ouvrage intitulé $L a$ Merveilleuse Aventure de Jacques Cartier. Cet ouvrage n'est pas une biographie, ni un essai historique. Il se présente comme un montage d'idées et de citations empruntant aux thèses d'Abel Lefranc (1905) qui a soutenu l'hypothèse d'une rencontre entre Rabelais et Jacques Cartier, de même qu'aux thèses de Jean Lefranc (1929), qui fait des voyages de Bougainville vers Tahiti le prolongement voire la reprise de ceux de Jacques Cartier en Amérique. Le présent article s'interroge sur la forme, les idées et la finalité poursuivies par Barbeau dans cet ouvrage qui n'a pas été retenu par sa postérité.
\end{abstract}

\begin{abstract}
In 1934, year marking the 400th anniversary of the discovery of Canada, Marius Barbeau, already known for his work as an ethnologist, publishes a book entitled The Wonderful Adventure by Jacques Cartier. This book is not a biography, nor a historical essay. It is presented as a montage of ideas and quotations borrowing from the theses of Abel Lefranc (1905) who supported the hypothesis of a meeting between Rabelais and Jacques Cartier, as well as the theses of Jean Lefranc (1929), who considers Bougainville's travels to Tahiti as the extension or even the resumption of those of Jacques Cartier in America. This article questions the form, ideas and purpose pursued by Barbeau in this book which was not retained by his posterity.
\end{abstract}


En 1934, paraît aux Éditions Albert Lévesque, dans la collection « Documents historiques », un petit ouvrage intitulé La Merveilleuse Aventure de Jacques Cartier, sous la signature de Marius Barbeau. Au moment où il publie cet ouvrage, Barbeau est anthropologue au Musée national du Canada. Le service où il travaille est un centre de recherches avancées dont le mandat est de « recueillir et sauvegarder les traditions culturelles des Amérindiens sous toutes leurs formes, organiser les expositions ethnographiques au Musée National et de présenter le fruit de ses recherches à la communauté scientifique et au grand public. » (Smith, 2003, p. 34) Ses méthodes de recherches « sont fondées sur le travail intensif sur le terrain » (Ibid., p. 34), dans la tradition des enquêtes prolongées auprès des dépositaires de ces traditions. Jean Du Berger parlera ainsi d'un « retour vers les conteurs » et vers les porteurs de la tradition (1976, p. 68). « L'entrée en fonction de Marius Barbeau comme anthropologue au Musée national d'Ottawa en 1911 a constitué un point tournant dans l'étude des traditions populaires du Canada français », écrit encore Jean-Pierre Pichette (2015, p. 373). Il y réalise ses études portant sur les Hurons-Wyandots, sur les Tsimshians de la côte nord-ouest de la Colombie britannique et sur les arts et traditions populaires du Canada français. Avant 1934, Marius Barbeau publie fréquemment ses travaux aux États-Unis par les soins de l'American Folklore Society, dans les mémoires de la Société Royale du Canada, à Toronto chez MacMillan, où paraissent surtout ses articles et ouvrages traitant des sociétés amérindiennes 
www.revue-analyses.org, vol. 15, nº1, printemps-été 2020, p. 73-93.

(wendat et de l'Ouest) ainsi que les contes et chansons qu'il recueille au cours de ses enquêtes. Il a obtenu deux fois le prix David (en 1925 et en 1929).

Si l'auteur est réputé dans le milieu des folkloristes, peut-être même parmi les écrivains, il n'est cependant pas une figure connue du grand public. Bien sûr, il a déjà, à cette date, contribué à la création des Veillées du bon vieux temps sous les auspices de la Société historique de Montréal et de la Société Saint-Jean-Baptiste (1919) puis à l'organisation du premier festival de folklore organisé par le Canadien Pacifique (1927), deux séries d'événements destinés au grand public, mais ce sont d'autres que lui - le chanteur Charles Marchand, par exemple - qui occupent l'avant-scène de ces événements. En effet, Barbeau n'a publié aucun livre en français avant La Merveilleuse Aventure de Jacques Cartier, qui suit tout juste la parution en anglais et à Toronto de son étude sur Cornelius Kriefhoff, l'année précédente, et qui accompagne cette année-là la publication de la collection d'articles qu'est Au cour de Québec. L'année suivante, il publiera deux recueils de contes chez Beauchemin (Grand'mère raconte et Il était une fois). Les œuvres littéraires plus connues de Barbeau, Romancero du Canada et Le rêve de Kamalmouk, paraîtront respectivement en 1937 et en 1948.

C'est donc avec cet ouvrage consacré à Jacques Cartier que Marius Barbeau inaugure une carrière littéraire d'écrivain, d'abord parce qu'il choisit la langue française comme langue d'écriture et de publication, ensuite parce qu'il donne à ses ouvrages une facture accessible : un nombre de pages limité, un caractère d'imprimerie bien aéré, des illustrations. En septembre 1934 avait paru, en anglais, dans la revue Canadian Geographical Journal, un premier article intitulé « Cartier Inspired Rabelais », dont est visiblement tiré l'extrait de $L a$ Merveilleuse Aventure de Jacques Cartier qui paraît en novembre 1934, dans La Revue 
populaire. Le mois suivant, la même revue publie la seule recension un peu substantielle, mais anonyme, de l'ouvrage. On y lit :

Cet ouvrage, qui fait de larges emprunts aux relations de Cartier et du Père Charlevoix renferme aussi de curieux extraits de Rabelais. Celui-ci, comme chacun sait, connut assez intimement Jacques Cartier. Il était venu chercher auprès du grand navigateur l'initiation à la science difficile de la navigation, dont il devait se servir dans le récit des pittoresques voyages de Pantagruel. M. Barbeau nous montre les corrélations manifestes des fantastiques récits de Rabelais avec les écrits et surtout les propos de Cartier. (Anonyme, 1934a, p. 27)

À première vue, la figure de Jacques Cartier, en tant qu'objet d'études, paraît bien éloignée des préoccupations scientifiques de Barbeau. L'année 1934 présente cependant l'occasion de s'y intéresser, car on célèbre cette année-là le $400^{\mathrm{e}}$ anniversaire de l'arrivée de Jacques Cartier à Gaspé. La commémoration semble propice à la redécouverte du personnage et l'on s'attendrait à voir paraitre plusieurs études ou biographies concurrentes à celle de Barbeau. La réalité est pourtant autre. Il faut dire que, en 1934, on célèbre bien des choses : le tricentenaire de la fondation de Trois-Rivières; le premier centenaire de la St. Patrick's Society of Montreal et le premier centenaire de la fondation de la Société Saint-Jean-Baptiste par Ludger Duvernay, tous événements fortement médiatisés dans l'espace public. Et, à vrai dire, hors des programmes des diverses célébrations, on note assez peu de travaux sur Cartier : citons tout de même la réédition de la biographie que lui avait consacrée NarcisseEutrope Dionne, un récit anecdotique sur la vie, les voyages et les aventures du Capitaine malouin par Joseph Dumais, un recueil de causeries historiques prononcées par l'abbé Victor Tremblay (Cartier a quand même consacré pas mal d'espace au royaume du Saguenay), une biographie destinée aux jeunes publics par Adélard Desrosiers. Ce serait bien mince s'il n’y avait concurremment la publication de l'ouvrage de Lionel Groulx, La découverte du Canada français. Jacques Cartier. 
www.revue-analyses.org, vol. 15, nº1, printemps-été 2020, p. 73-93.

\section{Une biographie romancée?}

Tel qu'il est formulé, le titre de l'ouvrage de Barbeau emprunte à première vue à la dynamique de la biographie romancée alors très à la mode et pour laquelle Albert Lévesque a conçu à la fois un concours (1933) et une collection baptisée « Figures canadiennes ». Henriette Tassé avait été la lauréate du concours pour son ouvrage La Vie humoristique d'Hector Berthelot, paru dans cette collection en 1934. Le titre du livre de Tassé n'a cependant pas tout à fait la même structure que celui de Barbeau. Celui-ci, La Merveilleuse Aventure de Jacques Cartier, est plus proche de quelques autres titres parus dans la même collection, notamment de La Vie aventureuse d'Arthur Buies de Raymond Douville (1933) et, plus encore, de La Grande Aventure de Le Moyne d'Iberville (1934) de Pierre Daviault. L'on s'attendrait ainsi à ce que l'ouvrage de Barbeau renvoie d'une manière ou d'une autre à ce type de biographie et peut-être même à une biographie romancée, genre dont il se rapproche tant par le titre (et à sa référence à la vie comme aventure) que par le format (qui est de moins de 150 pages). Tel n'est pourtant pas le cas.

L’ouvrage de Barbeau paraît plutôt dans la collection « Documents historiques », collection créée par Albert Lévesque en 1928, parmi les seize nouvelles collections que l'éditeur présente cette année-là à la Semaine du livre canadien et l'une des huit séries de « Documents ». Parmi celles-ci on trouve les « Documents historiques », « Documents économiques », « Documents philosophiques », « Documents linguistiques » et « Documents sociaux», qui ont en commun de réunir « des ouvrages de vulgarisation consacrés à différents aspects de la vie au Canada français (art, droit, économie, langue, philosophie, politique, problèmes sociaux). » (Michon, 1999, p. 282) En 1928, Lionel Groulx dirige cette collection, mais on ignore s’il est encore là en 1934 (Michon, 1999, p. 283). Quoi 
qu'il en soit, autant que les sources permettent de l'observer, les «Documents » sont clairement sous la dépendance du réseau de l'Action canadienne-française. La même année que La Merveilleuse Aventure de Jacques Cartier, paraissent dans la même collection deux autres ouvrages, ceux de Gustave Lanctôt, Le Canada d'hier et d'aujourd'hui, et de Georges Langlois, Histoire de la population canadienne-française. Aucun des deux ne rappelle le type d'ouvrage auquel appartient La Merveilleuse Aventure de Jacques Cartier, qui n'a ni l'ampleur ni les prétentions historiographiques de ces ouvrages et qui ne connaitra pas leur réception critique, plus riche et plus variée.

À vrai dire, au moment de sa publication (et outre l'article cité de La Revue populaire), l'ouvrage passe à peu près inaperçu. Malgré la notoriété de son auteur, il est rarement cité et encore moins commenté dans les études sur Jacques Cartier (excepté Berthiaume 1975) et même dans celles qui portent sur Marius Barbeau (Gauthier 2011; Nowry 1995; Nurse 1997). Ainsi, dans The Hero and the Historians. Historiography and the Uses of Jacques Cartier (2010), Alan Gordon glisse rapidement sur l'ouvrage et se réfère plutôt à l'article du Canadian Geographical Journal, pourtant beaucoup plus bref. Contrairement à la plupart des ouvrages parus chez Albert Lévesque, celui-ci se trouve rarement dans les bibliothèques publiques ou universitaires. L'exemplaire de la bibliothèque de l'Université du Québec à Montréal que j'ai consulté était à peine découpé. L'exemplaire de l'Université Laval (qui n'en a qu'un seul) fait partie de la collection Luc-Lacourcière et n'est donc accessible qu'en consultation sur place. Les vedettes-matières, qui servent à indexer l'ouvrage pour la consultation, restent vagues partout. On trouve: Nouvelle-France, Jacques Cartier, découverte et exploration. À l'occasion, on trouvera: voyage. Aucune bibliothèque ne fournit d'indication quant au genre littéraire ou de discours auquel appartiendrait le livre de 
www.revue-analyses.org, vol. 15, nº1, printemps-été 2020, p. 73-93.

Marius Barbeau. Les instruments de recherche livrent vraiment très peu de renseignements sur son contenu. Il faut dire que celui-ci a été publié sans paratexte autre que la page de titre; aucune préface ou introduction n'énonce le projet du livre et l'intention de l'auteur ou n'indique le ou les modèles dont il pourrait s'être inspiré.

Des éléments relevant d'une biographie de Jacques Cartier, il y aura dans l'ouvrage de Barbeau, mais des éléments seulement, et l'association aux titres semblables de la collection «Figures canadiennes», qui ferait de La Merveilleuse Aventure de Jacques Cartier une biographie romancée, apparaît rapidement illusoire. L'on reste cependant tout aussi songeur devant le nom de la collection « Documents historiques », car l'ouvrage de Marius Barbeau ne fait jamais appel à des sources manuscrites ou à des fonds d'archives. On n'y trouve pas de bibliographie, de notes infrapaginales, de critique des sources et autres éléments de méthodologie historiographique, ce qui est tout de même un peu étrange de la part d'un scientifique aussi chevronné. Il faut donc partir à zéro.

\section{Jacques Cartier et Rabelais}

La Merveilleuse Aventure de Jacques Cartier est un ouvrage divisé en trois parties de longueurs à peu près égales. La première partie du livre, « Blancs et Peaux-Rouges », porte sur l'arrivée des Blancs en Amérique. Elle ouvre sur une parole amérindienne, qui est une prophétie annonçant les dangers de la présence européenne. Puis elle fait intervenir la parole de Jacques Cartier par de larges extraits des relations ${ }^{1}$, incluant la relation du troisième voyage en 1541, «qui, rappelle Barbeau, est perdue, mais dont il subsiste une ancienne

\footnotetext{
${ }^{1}$ La première édition du Brief Récit de Jacques Cartier paraît à Paris en 1545. Pour l'ensemble des éditions et pour une discussion de leur authenticité, on consultera l'édition critique de Michel Bideaux (1986). Bideaux rend compte des hypothèses concernant les récits de Rabelais, mais il ne cite pas et ne commente pas le présent ouvrage de Marius Barbeau.
} 
traduction anglaise $[\ldots] .{ }^{2} \gg$ À la fin de cette partie, une troisième parole se fait entendre, celle de Roberval, qui était du voyage de 1541.

La deuxième partie, intitulée « Les mystères de la nature » ouvre sur le sort réservé aux Amérindiens que Cartier aurait emmenés en France. La parole est alors relayée à François Rabelais et aux voyages de Pantagruel ${ }^{3}$ en Andouille : «Rabelais, dans son Pantagruel, parodia ainsi l'incident des Peaux-Rouges » $(M A J C$, p. 41), affirme Barbeau, qui fait de cet épisode le premier où

il est question de l'impression que les naturels capturés aux antipodes [et donc pas seulement en Canada] firent à Paris ou que Paris fit sur eux, sauf en quelques récits de missionnaires ou dans le cas analogue des «tribulations d'Aoutourou », le Tahitien, qui avait suivi Bougainville en France. (MAJC, p. 42-43)

Cet épisode relatif à Aoutourou, qui établit une équivalence entre les voyages de Cartier en Canada et ceux de Bougainville à Tahiti, est une prolepse qui rapproche les deux siècles. Barbeau s'autorise ici des travaux d'Abel Lefranc qui, dans son ouvrage intitulé Les Voyages de Pantagruel, affirmait que Rabelais, contemporain de Cartier, aurait été son disciple quelque temps et qu'il en aurait appris les termes de marine et de pilotage, avant de faire de Cartier un personnage de son œuvre. Entre Cartier et Pantagruel, comme le suggère Abel Lefranc, il y a donc analogie voire « filiation d'idées qui rattache[nt] étroitement l'œuvre littéraire au fait historique et jette[nt] sur les deux une commune lumière. » (Lefranc, 1987 [1905], p. 51) Barbeau poursuit en reprenant d'autres extraits de l'étude de Lefranc pour démontrer que le pilote de Pantagruel, Jamet Brayet ou Brayer, est en réalité Jacques Cartier, et que le personnage de Xenomanes est en réalité, Jean Alfonse, un pilote cartographe

\footnotetext{
${ }^{2}$ Marius Barbeau, La merveilleuse aventure de Jacques Cartier, Montréal, Éditions Albert Lévesque, coll. «Documents historiques ", 1934, p. 34. Désormais, les références à cet ouvrage seront indiquées entre parenthèses par le signe $M A J C$, suivi du folio.

${ }^{3}$ Le Quart Livre de François Rabelais paraît à Paris en 1552.
} 
www.revue-analyses.org, vol. 15, nº1, printemps-été 2020, p. 73-93.

contemporain de Cartier, qui a parcouru les mers orientales. L'un et l'autre, dans leur version fictive, conduisent les expéditions de Pantagruel vers des terres promises et des trésors situés en Orient autant qu'en Amérique. «Ce [...] que Cartier, malgré ses efforts, n’arriva jamais à trouver, Rabelais le découvrit imaginativement et le parcourut à pleines voiles [...]. » (MAJC, p. 61)

Dans la troisième partie, intitulée « Pour quelques arpents de neige », Barbeau revient aux voyages de Cartier, à propos des descriptions de l'hiver et des maladies afférentes, notamment le scorbut. À l'extrait des voyages de Cartier, intitulé « Parmi les glaces et les neiges » $(M A J C$, p. 83), répond un autre extrait de Rabelais, intitulé « Les paroles gelées » (MAJC, p. 86). Suivent d'autres extraits empruntés à l'Histoire et description générale de la Nouvelle-France du père Charlevoix, qui permettent de raconter la préparation du troisième voyage de Cartier avec Roberval puis la suite des voyages vers la Nouvelle-France qu'ont menés De la Roche, Pontgravé, de Monts et Champlain. La fin de l'ouvrage nous projette de nouveau vers Bougainville, le contemporain de Voltaire, à qui Barbeau avait emprunté le titre de cette partie («Pour quelques arpents de neige»). Barbeau rappelle alors que Bougainville arrive au Canada en 1756 comme officier de Montcalm et qu'il s'ennuie au milieu des intrigues menées par Vaudreuil. Un extrait du livre de Jean Lefranc, Bougainville et ses compagnons, lui fait écrire :

Tout en notant ses observations sur le pays, les sauvages et leur langue, [Bougainville] lit des auteurs latins et grecs. [...] En vérité, dit Bougainville, les sauvages sont à peu de chose près les Grecs d'Homère. J'ai retrouvé Achille, Ajax, Ulysse, Nestor, Calchas, tout crachés. Nous avons bien aussi quelques Briséis [...]. (MAJC, p. 106)

C'est ce même Bougainville qui partira vers l'océan Pacifique, où il accoste « aux îles heureuses de la Nouvelle-Cythère » (MAJC, p. 110), aujourd'hui Tahiti. C'est dans cette 
partie que se trouve expliquée la notion de «merveilleuse aventure », conçue comme une chimère, un rêve poursuivi par la France tout entière « à la suite de Cartier » (MAJC, p. 108), rêve qui s'achève dans « [1]a désillusion et les revers politiques, en aboutissant à la Conquête du Canada par l'Angleterre. » (Ibid.)

\section{De Jacques Cartier à Bougainville}

Sur les 115 pages que contient le livre, une douzaine tout au plus sont de la main de Barbeau. Car le mode de composition est particulier : il s'agit d'un collage de citations. Sont utilisés, comme sources premières, des extraits des voyages de Jacques Cartier (y compris la relation de Roberval), le Quart livre de Rabelais et le Voyage autour du monde de Bougainville. Comme sources secondes, Barbeau reproduit des extraits de Charlevoix, d'Abel Lefranc, dont Les Navigations de Pantagruel ont paru à Paris en 1905, de Jean Lefranc, Bougainville et ses compagnons, ouvrage paru aussi à Paris en 1929. Il en résulte un montage, où domine le récit des voyages d'exploration, mais où les épisodes sont découpés par des paragraphes qui synthétisent les arguments des uns et des autres. C'est donc dans ce montage que réside la parole de Marius Barbeau. Or, ce montage présente quelques traits singuliers.

Le premier de ces traits singuliers est que l'ouvrage ouvre et ferme sur la parole des autochtones, les Amérindiens, conscients du danger que peut représenter la présence des Blancs en Amérique, alors que les Polynésiens auraient été plus sereins à l'arrivée des Blancs à Tahiti. Dans l'étude qu'il consacre à ce qu'il appelle «le couple mythique », formé de Jacques Cartier et l'Amérindien, André Berthiaume affirme que «Marius Barbeau est probablement le premier auteur, si l'on excepte l'abbé Jean-Baptiste-Antoine Ferland, à présenter avec sympathie le point de vue des autochtones » $(1975$, p. 88$)$ et, de surcroît, à 
www.revue-analyses.org, vol. 15, nº1, printemps-été 2020, p. 73-93.

« donner le point de vue des indigènes avant celui des Blancs : l'arrivée des Français, en 1534, est d'abord décrite dans la perspective des Hurons qui comparent la grande barque des Blancs à un Oiseau-Tonnerre descendu des nues. »(Ibid., p. 89) Berthiaume remarque également que tous les textes de Cartier qui racontent les entrevues qu'il a menées avec les Amérindiens sont d'une manière ou d'une autre cités par Barbeau. Il est probable, en effet, que Barbeau ait pris connaissance et travaillé en profondeur les récits de Cartier à travers ses études sur les Amérindiens et en préparation des études linguistiques qu'il va mener sur les langues huronne et iroquoienne, qui paraissent sous diverses formes entre 1915 et 1960.

Le deuxième trait singulier se trouve dans cette curieuse juxtaposition des figures de Cartier et de Bougainville que deux siècles séparent pourtant. L'un et l'autre ont en commun une relation particulière aux voyages d'exploration, qu'ils ont entrepris au service de l'État, découvrant tous deux une terre nouvelle que cet État va annexer. Lorsqu'elle est superposée aux voyages de Pantagruel, cette juxtaposition entraîne la réflexion vers l'utopie. En effet, dans Le Quart Livre, Rabelais représente le Canada comme une île. Claude La Charité rappelle que cette « représentation du Canada comme une île est usuelle à l'époque » (2011, p. 96) - on la retrouve chez Rabelais, mais aussi chez Marguerite de Navarre -, de sorte que les voyages de Pantagruel aboutissent en Utopie (avec la majuscule), - nom que Rabelais aurait emprunté à Thomas More selon Lefranc (1967 [1905], p. 30) -, mais dans une Utopie qui déçoit. Barbeau relève ainsi « la brutale réalité qui rebuta Pantagruel à l'Isle farouche » (MAJC, p. 95), qu'il associe au désenchantement de Cartier. Celui-ci, ayant renoncé à poursuivre ses voyages, se retire alors dans « une certaine obscurité » (MAJC, p. 108). C'est également dans une île, la Nouvelle-Cythère, qu'il représente comme un nouvel Éden, que débarque Bougainville. Celui-ci, écrit Barbeau, prend la relève de Cartier en ouvrant vers les 
mers du sud une nouvelle utopie, mais il parvient à « conjur[er] la vision [dysphorique] du Millénaire, déjà évoquée par Cartier et par Rabelais ». (MAJC, p. 112)

Le troisième trait singulier offert par l'écriture de Barbeau est dans la médiation qu'opèrent les ouvrages d'Abel Lefranc entre Cartier et Rabelais et de Jean Lefranc entre Bougainville et les philosophes des Lumières, Voltaire et Rousseau en particulier. Barbeau ne s'explique pas sur le choix de ces ouvrages ni même sur la manière dont il en a pris connaissance, mais les exemplaires des ouvrages d'Abel et Jean Lefranc qui sont conservés à la Collection nationale de Bibliothèque et Archives nationales du Québec proviennent de la collection Saint-Sulpice, ce qu'indiquent les ex-libris, bien que ces deux ouvrages soient français et qu'ils aient été publiés à Paris en leur temps respectif. Ces deux ouvrages étaient donc disponibles à la consultation à Montréal, mais sans doute aussi à la Bibliothèque du Parlement à Ottawa, au moment où Marius Barbeau écrivait son ouvrage. Ils agissent comme sources d'information et ils masquent ou travestissent la pensée de Barbeau, qui n'a donc plus à prendre position en son nom propre. Celui-ci se fait discret, intervient le moins possible, laisse parler les autres. Au début, la parole est aux Amérindiens puis elle est transmise aux universitaires européens, chaque groupe étant coiffé de son autorité propre.

\section{Utopie ou aventure?}

Il reste néanmoins à saisir le sens du titre et, de là peut-être, la fonction de l'ouvrage. Dans l'ouvrage de Barbeau, on trouve quelques occurrences de la locution « merveilleuse aventure » qui forme le titre. Ainsi, la phrase suivante : «La pensée française, emboîtant le pas après Cartier, Roberval et Rabelais, depuis François $1^{\text {er }}$ à la Révolution, se partagea en deux parties entre le beau rêve que suscita la Merveilleuse Aventure et la brutale réalité qui rebuta Pantagruel à l'Isle farouche. » $(M A J C$, p. 95) En effet, des trois représentations du 
www.revue-analyses.org, vol. 15, nº1, printemps-été 2020, p. 73-93.

Nouveau Monde étudiées par Robert Melançon dans la littérature française du XVI ${ }^{\mathrm{e}}$ siècle un paradis luxuriant, un monde propice aux prodiges et un désert stérile - c'est cette troisième interprétation qui s'imposera au lecteur européen à partir de l'échec de Roberval. D'autres occurrences viendront confirmer que cette « merveilleuse aventure » est un trait de la pensée politique qui, de la Renaissance à la guerre de Sept Ans, a entraîné les explorateurs à affronter les hasards d'une navigation ambitieuse, mal informée, livrée aux aléas de la faveur divine ou à une chimère qui, à la longue ne peut conduire qu'à un échec. Aussi, quand le dernier chapitre de l'ouvrage de Barbeau s'intitule «Vers l'utopie », c'est pour mieux revenir à Bougainville qui, après avoir été l'aide de camp de Montcalm en Nouvelle-France et après avoir subi la désillusion et les revers militaires qui aboutissent à la Conquête du Canada par l'Angleterre ${ }^{4}$ prend la relève de cette merveilleuse aventure en ouvrant vers les mers du sud une nouvelle navigation, informée des avancées scientifiques accomplies par la philosophie des Lumières. « L'utopie que Cartier croyait découvrir au royaume fabuleux du Saguenay ne continuait pas moins d'exister, mais ailleurs. » (MAJC, p. 110)

Cette «merveilleuse aventure » peut donc être assimilée à un moyen conceptuel capable de saisir le mode de connaissance empirique de l'univers. Rapprochant les deux textes, celui de Cartier et celui de Rabelais, Jean-Philippe Beaulieu explique ainsi que

les descriptions du Brief Recit sont organisées de façon épistémologique, tandis que celles du Quart Livre le sont de manière rhétorique, c'est-à-dire que les descriptions de Cartier se préoccupent surtout de mettre en valeur le signifié, c'est-à-dire l'information, tandis que celles de Rabelais accordent une importance particulière au signifiant, c'està-dire aux différents procédés de présentation de l'information. (Beaulieu, 1985, p. 105)

\footnotetext{
${ }^{4}$ On lit ainsi : «La désillusion et les revers politiques, en aboutissant à la Conquête du Canada par l'Angleterre, achevèrent la merveilleuse aventure où la France s'était engagée à la suite de Jacques Cartier. » (MAJC, p. 108)
} 
Son analyse l'amène à dissocier les modes de pensée propres à Cartier et à Rabelais. Il conclut que les récits de voyage de Jacques Cartier font preuve d'une orientation intellectuelle « renaissante », alors que les descriptions de Rabelais relèvent d'une sorte de théocentrisme qui agit comme principe unificateur. La « merveilleuse aventure » serait donc cette ouverture renaissante alors que l'utopie rabelaisienne relèverait d'une épistémè encore médiévale bien qu'en fin de parcours. On pourrait sans doute lire de la même manière le Voyage autour du monde de Bougainville (1771), car si Cartier est relu par Rabelais, Bougainville sera lui relu par les Philosophes du XVIII ${ }^{\mathrm{e}}$ siècle, notamment par Denis Diderot, qui publie un assez révisionniste Supplément aux Voyages de Bougainville (1796). Ainsi Barbeau fait-il de Bougainville le porteur de la pensée philosophique nouvelle au XVIII ${ }^{\mathrm{e}}$ siècle, car la Nouvelle-Cythère est d'abord l'utopie de ces philosophes, avant d'être la sienne propre : « Jean-Jacques Rousseau s'épancha dans d'éloquentes déclamations contre la civilisation. [...] Diderot la voyait habitée par la justice et Voltaire par la sagesse... » (MAJC, p. 113) De ce point de vue, les voyages contribuent à déboulonner l'avenir, à ouvrir vers des horizons inconnus, là où l'utopie ne peut faire autrement que renverser les points de vue anciens sur eux-mêmes sans jamais parvenir à les dépasser entièrement. La chute de l'ouvrage repose sur une ellipse révélatrice : « Déjà poignait à l'horizon la devise humanitaire et utopique : Liberté, Égalité, Fraternité! »(MAJC, p. 113)

\section{Un ouvrage polémique?}

Revenons maintenant à Lionel Groulx. La même année que Barbeau, Lionel Groulx consacre lui aussi un livre à Jacques Cartier. C'est à la demande de ses collaborateurs et à partir de ses notes de cours que Lionel Groulx publie La Découverte du Canada. Jacques Cartier. Il écrit : « Mais nous voici au quatrième centenaire de la découverte du Canda. Peut- 
www.revue-analyses.org, vol. 15, nº1, printemps-été 2020, p. 73-93.

être serait-il opportun, m'a-t-on représenté, que les Canadiens eussent l'air de s'en apercevoir. » (1934, p. 9) Malgré son titre, qui adjoint le nom propre à l'événement, et de ce fait laisse présager des éléments de biographie, Groulx propose un ouvrage sur l'expansion européenne en Amérique, qu'il conçoit comme un chapitre préliminaire de l'histoire du Canada $^{5}$. L'ouvrage, divisé en six chapitres, aborde successivement la découverte du Nouveau Monde, de l'Amérique du Nord et du Canada, insistant sur le travail d'exploration mené par Jacques Cartier au cours de ses trois voyages. Il s'agit d' « un bien curieux livre » écrit André Berthiaume qui observe, chez Groulx, une conception très orthodoxe de la Renaissance, qui aurait mis «à la mode un humanisme païen» et provoqué « une décomposition moléculaire de la chrétienté ». Dans ce « désordre apocalyptique », la mission de Cartier apparaît «comme une édifiante épopée ». (Berthiaume, 1975, p. 86) Sur les Amérindiens, Groulx ressasse les vieux poncifs : les indigènes sont de «grands enfants versatiles », hypocrites, naïfs, envieux, cupides. En un mot, ils sont barbares. (Ibid., p. 87) À la différence de l'ouvrage de Barbeau toutefois, celui de Groulx repose sur documentation riche et de première main, sérieusement discutée, et utilisée avec discernement. Il émet plusieurs observations pertinentes sur l'authenticité des récits de Cartier, sur l'époque, etc. Bref le travail de Groulx est un travail d'historien professionnel. Rien de cela chez Marius Barbeau qui convoque des écrivains, des critiques littéraires, des historiens de la littérature, les philosophes des Lumières pour construire sa propre lecture de l'événement et qui, de ce fait, n'affronte jamais Groulx sur le terrain de l'historiographie. Historien contre ethnologue serait déjà une manière de caractériser la relation entre ces deux livres. Ajoutons que les

\footnotetext{
${ }^{5}$ « On ne conçoit point une histoire du Canada sans un chapitre préliminaire sur les découvertes. » (Groulx, 1934, p. 13)
} 
travaux d'Abel Lefranc ont en leur temps affronté de solides critiques : son hypothèse concernant l'éventuelle rencontre entre Cartier et Rabelais a été maintes fois discutée et remise en question par des historiens qui ont tenté des vérifications factuelles et n'ont trouvé que de douteux témoignages (Bideaux 1986) et sans doute que la fragilité voire les réfutations de la preuve historiographique avancée par Barbeau ont été cause de l'indifférence qui entoure encore aujourd'hui cet ouvrage.

Les critiques et réfutations sont cependant toutes ultérieures à la publication de l'ouvrage de Marius Barbeau et peut-être que, en insistant sur les strictes dimensions historiographiques, l'on rate à la fois l'audace, l'imagination et l'ambition de Barbeau. Peutêtre faut-il après tout revenir à l'hypothèse sinon d'une biographie du moins d'une histoire romancée, où les raccourcis, même abusifs, de la narration engendrent une argumentation nouvelle, télescopant les époques pour juxtaposer les idées, pour témoigner de la volonté, commune aux explorateurs, de se projeter dans l'inconnu pour y saisir l'occasion de réinventer l'avenir. André Berthiaume considère ainsi l'ouvrage de Barbeau comme un « tournant dans la fortune des récits de voyage de Cartier » parce qu'il s'écarte résolument de la tradition « romantico-patriotarde »-1'expression est de lui - pour « mettre l'accent sur l'humanité des indigènes et les résonances intellectuelles (et politiques) des voyages d'exploration. » (Berthiaume, 1975, p. 90) Nous ne sommes pas loin d'une forme d'histoire qu'on appellera l'histoire des idées, précisément située à la jonction entre l'histoire de la philosophie, l'histoire des sciences et l'histoire littéraire. Ce n'est pourtant que dix ans plus tard, en 1944, que Lucien Febvre publiera Autour de l'Heptaméron. Amour sacré, amour profane. Mais je ne vois pas d'autre manière de conclure que celle d'affirmer que $L a$ 
www.revue-analyses.org, vol. 15, nº1, printemps-été 2020, p. 73-93.

Merveilleuse Aventure de Jacques Cartier s'inscrit dans l'archéologie de cette histoire des idées et que Marius Barbeau a trouvé là, le moyen de proposer, sans doute pas une réponse la publication des deux ouvrages est trop proche dans le temps -, mais néanmoins une contrelecture à La Découverte du Canada telle que la concevait Lionel Groulx. De ce point de vue, Barbeau paraît mû davantage par les résonances intellectuelles et politiques des voyages d'exploration que par la genèse de l'identité nationale, qui reste l'objectif ultime de Groulx, lequel, depuis les années 1920, dans ses cours et conférences à 1'Université de Montréal ou dans les pages de la revue L'Action française puis L'Action canadienne-française s'est imposé comme maître à penser. Peu de voix se sont opposées à la réflexion savante sur la société québécoise, néanmoins orientée vers une rhétorique de l'adhésion au programme social de l'Église catholique, que mènent les intellectuels de son entourage, tels Édouard Montpetit, Arthur Saint-Pierre, Hermas Bastien ou Esdras Minville. Comme la poésie exotiste qui s’opposait au régionalisme des années 1920, la pensée de Barbeau, pensée de résistance au nationalisme conservateur, refuse l'argumentation convenue et se déploie dans la liberté permise aux écritures qui font appel à l'imagination voire à la fiction, pour rappeler que la découverte du Canada a été rendue possible par cette révolution épistémologique qu'a été la Renaissance, à laquelle ont contribué diversement Rabelais et Cartier. C'est bien pour raviver le caractère révolutionnaire de la Renaissance que Barbeau lui superpose la découverte de Tahiti par Bougainville, elle-même inscrite dans un processus qui mène inexorablement vers la Révolution française, à la source des démocraties modernes. Il s’agit donc de raviver l'idée de «merveilleuse aventure », cette pensée renaissante qui se projette dans l'avenir, contre « notre maître le passé », selon le titre donné par Lionel Groulx à son 
recueil d'articles et de conférences (1924), chantre de la pensée médiévale et héraut de l'Ancien Régime aristocratique.

\section{Bibliographie}

Anonyme (1934a), «La Merveilleuse Aventure de Jacques Cartier », La Revue populaire, décembre, p. 27.

ANONYME (1934c), « Bibliographie canadienne », Le Terroir, vol. XVI, no 5, octobre, p. 910 .

Anonyme (1935), « Chronique du Canada français », La Renaissance, 2 novembre, p. 5.

BARBEAU, Marius (1915), Classification of Iroquoian Radicals with Subjective Prenominal Prefixes, Ottawa, Geological Survey of Canada Memoir nº 46, Anthropological Series, no 7.

BArbeAu, Marius (1934a), La Merveilleuse Aventure de Jacques Cartier, Montréal, Éditions Albert Lévesque, coll. « Documents historiques ». Un extrait parut dans La Revue moderne, novembre, p. 9.

Barbeau, Marius (1934b), Au cœur de Québec, Montréal, Les Éditions du Zodiaque et Librairie Déom frère.

Barbeau, Marius (1934c), Cornelius Krieghoff. Pioneer Painter of North America, Toronto, MacMillan.

BARBEAU, Marius (1934d), « Cartier inspired Rabelais », Canadian Geographical Journal, vol. IX, no 3, septembre, p. 113-125.

BARBEAU, Marius (1935), Grand'Mère raconte, illustrations de Phoebé Thomson, Montréal, Éditions Beauchemin.

BARBEAU, Marius (1935a), Il était une fois, illustrations de Phoébé Thomson, Montréal, Éditions Beauchemin.

Barbeau, Marius (1937), Romancero du Canada, préface de Marguerite Béclard d'Harcourt, Montréal, Éditions Beauchemin.

BARBEAU, Marius (1948), Le rêve de Kamalmouk, dessins de Grace Melvin, préface de Luc Lacourcière, Montréal, Fides, coll. « Nénuphar ». 
www.revue-analyses.org, vol. 15, $\mathrm{n}^{\circ} 1$, printemps-été 2020, p. 73-93.

Barbeau, Marius (1960), «The Language of Canada in the Voyages of Jacques Cartier (1534-1538) », Musée national du Canada. Bulletin, nº 173, p. 108-229.

BEAULIEU, Jean-Philippe (1985), « La description de la nouveauté dans les récits de voyage de Cartier et de Rabelais », Renaissance and Reformation, vol. IX, nº 2, p. 104-110.

BELlemare, Luc (2013), "Du théâtre en chanson? La transfolklorisation chez les collaborateurs du Carillon canadien de Charles Marchand», Les Cahiers de la Société québécoise de recherche en musique, vol. XIV, nº 2, p. 39-58.

Berthiaume, André (1975), «La fortune d'un couple mythique: Jacques Cartier et l'Amérindien », Études littéraires, vol. VIII, n 1, avril, p. 81-102.

BIDEAuX, Michel (1986), Brief Récit de Jacques Cartier, édition critique, Montréal, Presses de l’Université de Montréal, coll. «Bibliothèque du Nouveau Monde ».

BOUGAINVILLE, Louis-Antoine de (1771) Voyage autour du monde, par la frégate du roi La Boudeuse et la flûte L'Étoile; en 1766, 1767, 1768 \& 1769, Paris, Chez Saillant \& Nyon, libraires.

CharlevolX Pierre-François-Xavier de (1744), Histoire et description générale de la Nouvelle-France, Paris, Chez Rolin fils, libraire, 3 vol.

Daviault, Pierre (1934), La Grande Aventure de Le Moyne d'Iberville, Montréal, Éditions Albert Lévesque, coll. «Figures canadiennes ».

Desrosiers, Adélard (1934), Notre Jacques Cartier, Montréal, Éditions Albert Lévesque.

DiDEROT, Denis (1995 [1796]), Supplément au Voyage de Bougainville, Paris, Librairie générale française.

DionNe, Narcisse-Eutrope (1933 [1889]), Jacques Cartier, Québec, Imprimerie Émile Robitaille.

DouviLLE, Raymond (1933), La Vie aventureuse d'Arthur Buies, Montréal, Éditions Albert Lévesque, coll. « Figures canadiennes ».

DU BERGER, Jean (1976), «Marius Barbeau : le conte et le conteur », Études françaises, vol. XII, no 1-2, avril, p. 61-70.

DumaIS, Joseph (1934), Le Capitaine malouin Jacques Cartier, découvreur officiel du Canada; récit anecdotique de sa vie, de ses voyages et aventures, précédé de notes intéressantes sur les voyages antérieurs des navigateurs norvégiens et autres, Québec, en vente à la Fierté française. 
FEBVRE, Lucien (1944), Autour de l'Heptaméron. Amour sacré, amour profane, Paris, Gallimard.

GAUTHIER-LAROUCHE, Georges (2010), « Merveilleuse Aventure de Jacques Cartier (La) par Marius Barbeau », dans Gaétan Gervais et Jean-Pierre Pichette (dir.), Dictionnaire des écrits de l'Ontario français, 1613-1993, Ottawa, Les Presses de l'Université d'Ottawa, p. 535-536.

GroulX, Lionel (1934), La Découverte du Canada. Jacques Cartier, Montréal, Librairie Granger frères limitée.

GroulX, Lionel (1924), Notre maître, le passé [Première série], Montréal, Bibliothèque de l'Action française.

LA CHARITÉ, Claude (2011), « Les questions laissées en suspens par le Brief Recit (1545) de Jacques Cartier et les réponses de la nouvelle 67 de L'Heptaméron (1559) de Marguerite de Navarre », Euvres et critiques, vol. XXXVI, n 1, p. 91-109.

LANCTÔT, Gustave (1934), Le Canada d'hier et d'aujourd'hui, Montréal, Albert Lévesque, coll. « Documents historiques ».

LANGLOIS, Georges (1934), Histoire de la population canadienne-française, Montréal, Albert Lévesque, coll. « Documents historiques ».

LeFrANC, Abel (1967 [1905]), Les Navigations de Pantagruel. Étude sur la géographie rabelaisienne, Paris, réédition Slatkine.

LEFRANC, Jean (1929), Bougainville et ses compagnons, Paris, Albin Michel, coll. « Les vies authentiques $\gg$.

MElAnÇON, Robert (1979), «Terre de Caïn, âge d'or, prodiges du Saguenay: représentations du Nouveau Monde dans les voyages de Jacques Cartier », Studies in Canadian Literature, vol. IV, $\mathrm{n}^{\mathrm{o}}$ 2, été, p. 22-34.

Michon, Jacques (2010), L'Édition littéraire en quête d'autonomie. Albert Lévesque et son temps, Québec, Presses de l’Université Laval.

Michon, Jacques (dir.) (1999), Histoire de l'édition littéraire au Québec, tome I, La naissance de l'éditeur. 1900-1959, Montréal, Fides.

Nowry, Lawrence (1995), Marius Barbeau. Man of Mana. A Biography, Toronto, NC Press.

NuRSE, Andrew (1997), « Tradition and Modernity : The Cultural Work of Marius Barbeau ». Thèse de Ph. D., Kingston, Queen's University.

PICHETTE, Jean-Pierre (2015), « Marius Barbeau : au-delà du souvenir », Rabaska, vol. XIII, p. 372-378. 
www.revue-analyses.org, vol. 15, nº1, printemps-été 2020, p. 73-93.

SMITH, Gordon E. (2003), « Le discours sur la musique traditionnelle dans l'œuvre de Marius Barbeau », Les Cahiers de la Société québécoise de recherche en musique, vol. VII, nº 1-2, p. 33-42.

TASSÉ, Henriette (1934), La Vie humoristique d'Hector Berthelot, préface de Victor Morin, Montréal, Éditions Albert Lévesque, coll. « Figures canadiennes ».

TREMBlay, Victor (1934), Le Temps de Jacques Cartier; causeries historiques, Chicoutimi, Société historique du Saguenay.

\section{Notice biobibliographique}

Lucie Robert enseigne la littérature québécoise à l'Université du Québec à Montréal. Elle est membre du Centre de recherche interuniversitaire sur la littérature et la culture québécoises (CRILCQ), où elle codirige le collectif La vie littéraire au Québec, 1764-1947 (PUL, 6 vol. parus depuis 1991; vol. VII et VIII en préparation). Ses plus récents ouvrages s'intitulent Apprivoiser la modernité. La pièce en un acte de la Belle Époque à la Crise (Nota Bene 2012), Le théâtre en revue (PUQ 2014) en collaboration avec Shawn Huffman, et La littérature comme objet social II. Mélanges offerts à Denis Saint-Jacques (Nota bene 2019), en collaboration avec Marie-Andrée Beaudet et Micheline Cambron. Elle est membre de la Société des Dix. 